\title{
The Method of Tolerance Design and Verification of Parts Based on Comprehensive Geometry Precision of Five-axis Machine Tool
}

\author{
Li-Chang Zhu, Jun Wu, Ming Li, Qing-Yue Wei, Yang Yang, Li-Jia Ren \\ Shanghai Key Laboratory of Intelligent Manufacturing, and Robotics, \\ Shanghai University, \\ Shanghai, China \\ E-mail: zlconly@163.com, Shu_wujun@163.com,robotlib@shu.edu.cn,76328851@qq.com, sduyangyangs@163.com, \\ 893891338@qq.com
}

\begin{abstract}
According to the comprehensive geometric accuracy of machine tool in the given machine, calculated along the axial direction, the accuracy of parameters, and then optimize the distribution become more cutting edge precision machine tool design. However, comprehensive geometric accuracy of machine tools to ensure eventually to implement on the movement of parts tolerance. Of the defects in the design method of this article in view of the present machine tool accuracy, put forward a kind of based on the geometry precision of five-axis machine tools integrated parts of tolerance design and verification method.
\end{abstract}

Keywords-component; five-axis machine tool; geometric accuracy ; accuracy design; tolerance design

\section{INTRODUCTION}

For Five-axis machine tool, motion pairs are more, and kinematic chain is longer. The overall stiffness and accuracy are easy to be affected. Since more and more users is vitally strict with quality and precision of parts, the research how to ensure comprehensive geometric accuracy five-axis machine tools to guarantee precision of parts machining become urgent gradually.

In machine tool design application, how to ensure and improve the comprehensive geometric accuracy five-axis machine tools is a key technology that must be solved. Fiveaxis machine tool accuracy design is based on the given integrated geometric accuracy, calculated accuracy along the axis direction backward, and then optimized distribution; Through using parts that meet the requirements of the precision to ensure the accuracy of the machine along the axis direction of movement, and ensure that meet the requirements of comprehensive geometric accuracy of machine tools ultimately. With the appearance of the machine tool wear, the accuracy is also easy to lose. Therefore, the subsequent method of error compensation to ensure comprehensive machine tool geometric accuracy requirements can be used. Through collecting existing machine tool geometric error in the system, modeling mathematical model, and generating error model, then introduce a new error compensating system, namely in the original system to add a new correction term, the current error can be offset finally.

Therefore, when compensated for comprehensive error of five-axis machine tools, motion error along the axis direction of machine tools must be measured; it is difficult for machine tool error compensation actually. Since the tolerance is used to limit the error, comprehensive machine tool geometric error is solved according to the superposition of each pair of its various random geometry errors. So we cover geometric motion error of each axis by providing each motion pair parts geometric tolerance. Finally geometric tolerance analysis model of five-axis machine tool is established. Since comprehensive combination machine tool geometric error is random, we can makes comprehensive geometric accuracy of machine tool design landed on the specific composition geometric tolerance through utilizing Monte Carlo method for three dimensional error simulation analysis. In the meanwhile, using the contribution factor that affected the accuracy of machine tool integrated geometry, it can provide good evidence for the comprehensive error compensation subsequently.

\section{COMPREHENSIVE GEOMETRIC ACCURACY OF MACHINE TOOL DESIGN}

\section{A. Machine Tool Geometric Error Term}

For five axis machine tool, there are three parallel movements respectively along the $\mathrm{X}, \mathrm{Y}, \mathrm{Z}$ direction of three axis. Once changed, each axis will three line displacement error generate along the $\mathrm{X}, \mathrm{Y}, \mathrm{Z}$ direction, such as three angular displacement error around $\mathrm{X}, \mathrm{Y}$, and $\mathrm{Z}$ direction, three angular displacement error around $\mathrm{X}, \mathrm{Y}$, and $\mathrm{Z}$ direction. There are two axes $\mathrm{AB}$ or $(\mathrm{BC}, \mathrm{AC})$ along the $\mathrm{X}$, $\mathrm{Y}, \mathrm{Z}$ direction is similar. In addition, three linear motions will produce three perpendicularity between motion shaft directions. These three perpendicularity error does not change along with the movement of machine tool movement, so there are 33 items geometric direction error for five-axis machine tools totally.

As for translational axis, working platform of machine tool has six error - three displacement error and three Angle error along the guide rail movement. For example, there are three displacement error $\delta_{\mathrm{x}}(\mathrm{X}), \delta_{\mathrm{y}}(\mathrm{X}), \delta_{\mathrm{z}}(\mathrm{X})$ and three Angle error $\varepsilon_{\mathrm{X}}(\mathrm{X}), \varepsilon_{\mathrm{y}}(\mathrm{X}), \varepsilon_{\mathrm{z}}(\mathrm{X})$ along the $\mathrm{X}$ axis direction for translational direction of $\mathrm{X}$ axis. At this time, $\delta$ is on behalf of the displacement error, $\varepsilon$ is on behalf of the Angle error, the letters in brackets represent the movement direction, and subscript letter is on behalf of the error direction. 
Along the $\mathrm{Y}$ direction, translation error is: $\delta_{\mathrm{x}}(\mathrm{Y}), \delta_{\mathrm{y}}(\mathrm{Y})$, $\delta_{\mathrm{z}}(\mathrm{Y}), \varepsilon_{\mathrm{x}}(\mathrm{Y}), \varepsilon_{\mathrm{y}}(\mathrm{Y}), \varepsilon_{\mathrm{z}}(\mathrm{Y})$.

Along the $Z$ axis direction translation error is: $\delta_{\mathrm{x}}(\mathrm{Z}), \delta_{\mathrm{y}}(\mathrm{Z}), \delta_{\mathrm{z}}(\mathrm{Z}), \varepsilon_{\mathrm{x}}(\mathrm{Z}), \varepsilon_{\mathrm{y}}(\mathrm{Z}), \varepsilon_{\mathrm{z}}(\mathrm{Z})$.

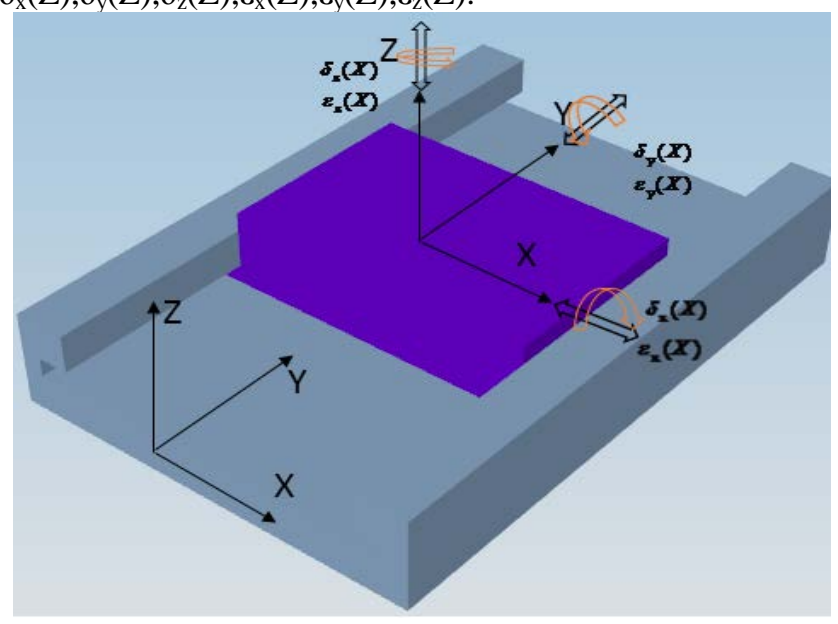

Figure 1. Translational axis machine tool geometric error.

\section{B. Parts of Motion Pair Geometric Tolerance Control}

Assume that $\mathrm{M}$ is a point of the worktable. Since the straightness error and angle error are exists, then motion trail of $\mathrm{M}$ point is shown in figure 2, you can see that it is still restricted within a certain limit of volatility, and do not exceed the limit. It is similar to the concept of profile error in size tolerance.( Fig. 3)

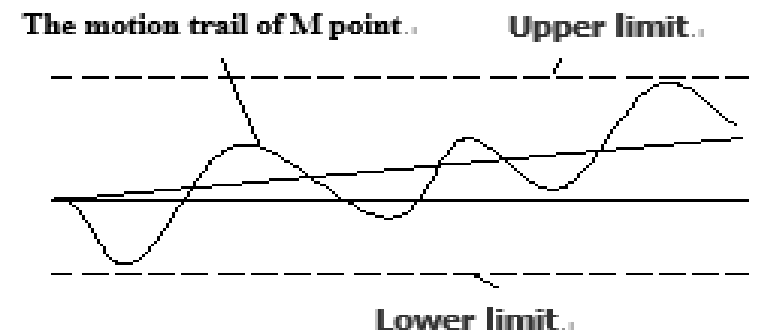

Figure 2. The motion trail of $\mathrm{M}$ point.

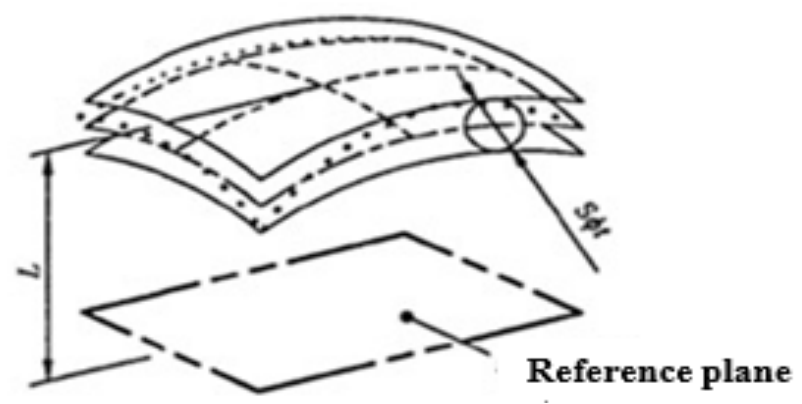

Figure 3. The concept of surface profile.
According to the analysis on the above, When slider move along the $\mathrm{X}$ axis direction, due to the existence of the guide rail straightness error and angle error, the workbench in the $\mathrm{XY}$ plane and the YZ plane will twist within a certain range. So in view of the flat guide, It can be converted into the face of the contact surface profile and verticality. The flatness of a given contact underside can cover $\delta_{\mathrm{y}}(\mathrm{X}), \varepsilon_{\mathrm{X}}$ and $\varepsilon_{\mathrm{z}}(\mathrm{X})$, two contact verticality can cover $\delta_{\mathrm{z}}(\mathrm{X})$ and $\varepsilon_{\mathrm{y}}(\mathrm{X})$; For cylindrical guide can be converted into cylindrical guide rail straightness and verticality. Given the location of the axis of the cylindrical guide can cover $\delta_{\mathrm{z}}(\mathrm{X}), \varepsilon_{\mathrm{X}}(\mathrm{X})$ and $\varepsilon_{\mathrm{y}}(\mathrm{X})$, vertical degree can cover $\delta_{\mathrm{y}}(\mathrm{X}), \varepsilon_{\mathrm{z}}(\mathrm{X})$; By the machine tool along the $\mathrm{X}$ axis server motor precision is limit by $\delta_{\mathrm{x}}(\mathrm{X})$. The surface profile and verticality of the initial value is as follows:

Factors that affect bottom flatness are: $\delta_{\mathrm{y}}(\mathrm{X}), \varepsilon_{\mathrm{x}}(\mathrm{X})$ and $\varepsilon_{y}(X)$,The size of the resulting flatness $\triangle_{\mathrm{a}}$, See the following formula (1):

$$
\Delta_{a}=\delta_{y}(X)+\sin \left(\varepsilon_{z}(X)\right) * \frac{L_{a x}}{2}+\sin \left(\varepsilon_{x}(X)\right) * \frac{L_{a y}}{2}
$$

Factors that affect bottom flatness are: $\delta_{z}(X)$ and $\varepsilon_{\mathrm{x}}(\mathrm{X})$,The size of the resulting flatness $\triangle_{\mathrm{b}}$, See the following formula (2):

$$
\Delta_{\mathrm{b}}=\delta_{\mathrm{z}}(X)+\sin \left(\varepsilon_{\mathrm{y}}(X)\right) * \frac{L_{\mathrm{ax}}}{2}
$$

$\mathrm{L}_{\mathrm{ax}}, \mathrm{L}_{\mathrm{ay}}$ represent the workbench and machine tool guide way joint surface respectively in the length of the $\mathrm{X}, \mathrm{Y}$ direction, Angle is arc system; Position to the axis of the cylindrical guide size analysis process is similar to the former. Therefore, parts of motion pair geometric tolerance model of machine tool are as follows:

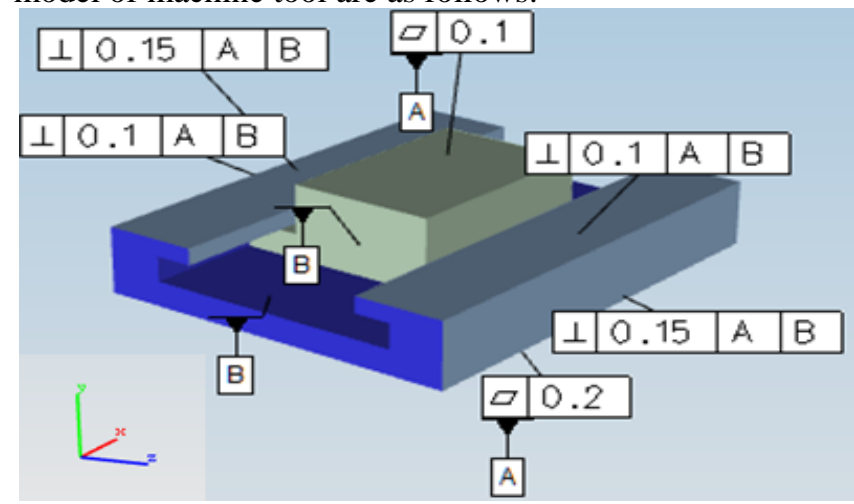

Figure 4. Tolerance of flat guide movement part. 


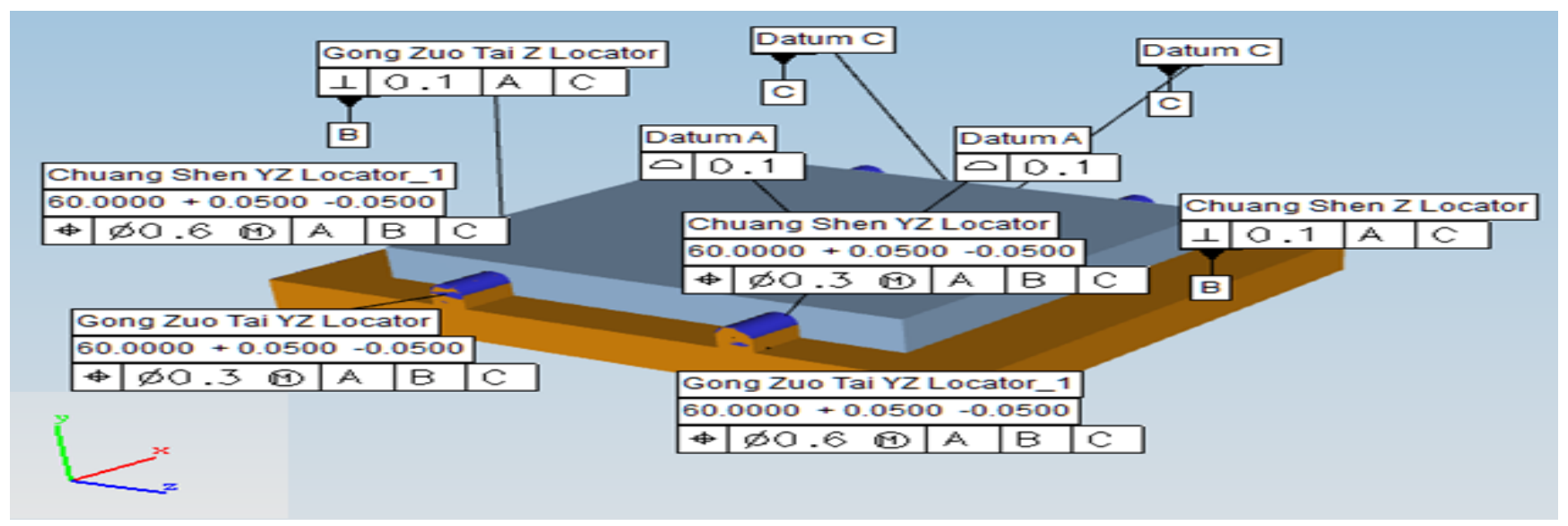

Figure 5. Tolerance of cylindrical guide moving parts.

\section{Set up Machine Tool Geometric Tolerance Model}

In computer aided tolerance simulation analysis software, dimension chain is geometric tolerance model, it is calculated by the superposition of geometric tolerance of various parts of motion pair. Thus when analyze integrated geometric error, it need to translate the various geometric error into the geometrical characteristics tolerance of the parts of machine assembly to solve.

In this Paper, we take TTTRR class of five axis machine tool as an example. In geometric tolerance analysis model of machine tool is set up, we just consider important parts (such as the moving parts), and ignore most of the intensive parts (such as standard parts, etc.). For parts of making up mobile pair, It need to provide flatness and perpendicularity of two parts assembly contact characteristics to limit the movement error along the moving direction; For component of making up cylindrical guide mobile pair, It need to provide position of cylindrical axis. For composition of making up rotation pair, It need to provide position and alignment of two parts assembly contact characteristics to limit the movement error along the rotation direction; Between the vertical errors and parallel errors of machine tool along the axis direction requires each assembly feature of tolerance and mutual flatness to constraints together. Through the transformation of geometric error term on the above, five-axis machine tool geometric tolerance analysis model is established according to actual machine tool assembly sequence (Fig. 6).

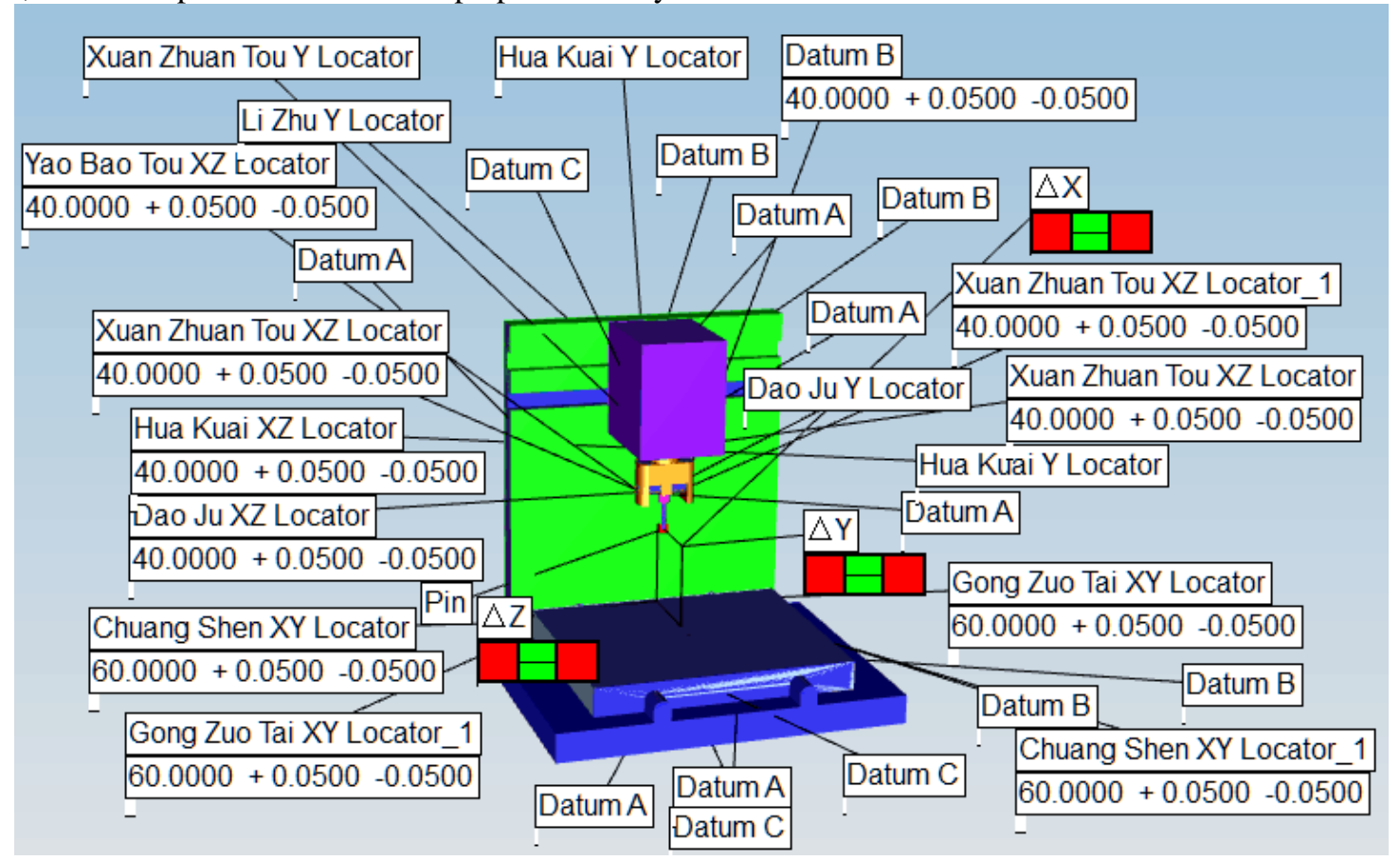

Figure 6. Machine tool geometric tolerance model. 


\section{ANALYSIS OF COMPREHENSIVE GEOMETRIC ERROR}

The common method of tolerance analysis have the extreme value method, the statistical tolerance method and Monte Carlo method.

The principle of the extreme method is simple, small amount of calculation, no need to consider the size distribution within the tolerance zone. It can guarantee the success rate and $100 \%$ of parts interchangeability. While this design is too conservative, and increase the processing cost. The method of statistical tolerance analysis is to describe the change of parts size at the form of a statistical distribution, and calculated the statistical distribution of the parts manufacturing capability and assemble function. However, this method need assumes that parts tolerance obey normal distribution, and have a linear relationship between tolerance and assembly parts tolerance. Actually, the result does not quite accord with the true situation. The Monte Carlo method is to take the closed loop dimension and tolerance as statistics of a random variable. According to the actual distribution of each size, to take a certain algorithm to sampling through the computer, generate the corresponding random numbers, and then calculate the closed loop dimension and tolerance according to design function. Under given conditions, the results that obtained through this method conform to the actual situation.

Considering the nature of geometric error of machine tool, it is formed of superimposing various random geometric error of each motion pair based on the structure of the machine tool. Therefore the comprehensive error of machine tool is random. The Monte Carlo method is also used to solve the problem of this kind of random. It is that use statistical methods of random variables to solve the problem of closed loop dimension and tolerance. Tolerance is in line with the random distribution, and is limited within a certain range of variables. So the properties are similar with geometric errors of machine tools. Therefore the Monte Carlo simulation method can be used to the analysis of 3D geometric error simulation for comprehensive geometric error of machine tool. There is a variety of mature tolerances simulation software using Monte Carlo method. VISVSA, developed by Siemens Company, is mainly used in calculation of space dimension chain.

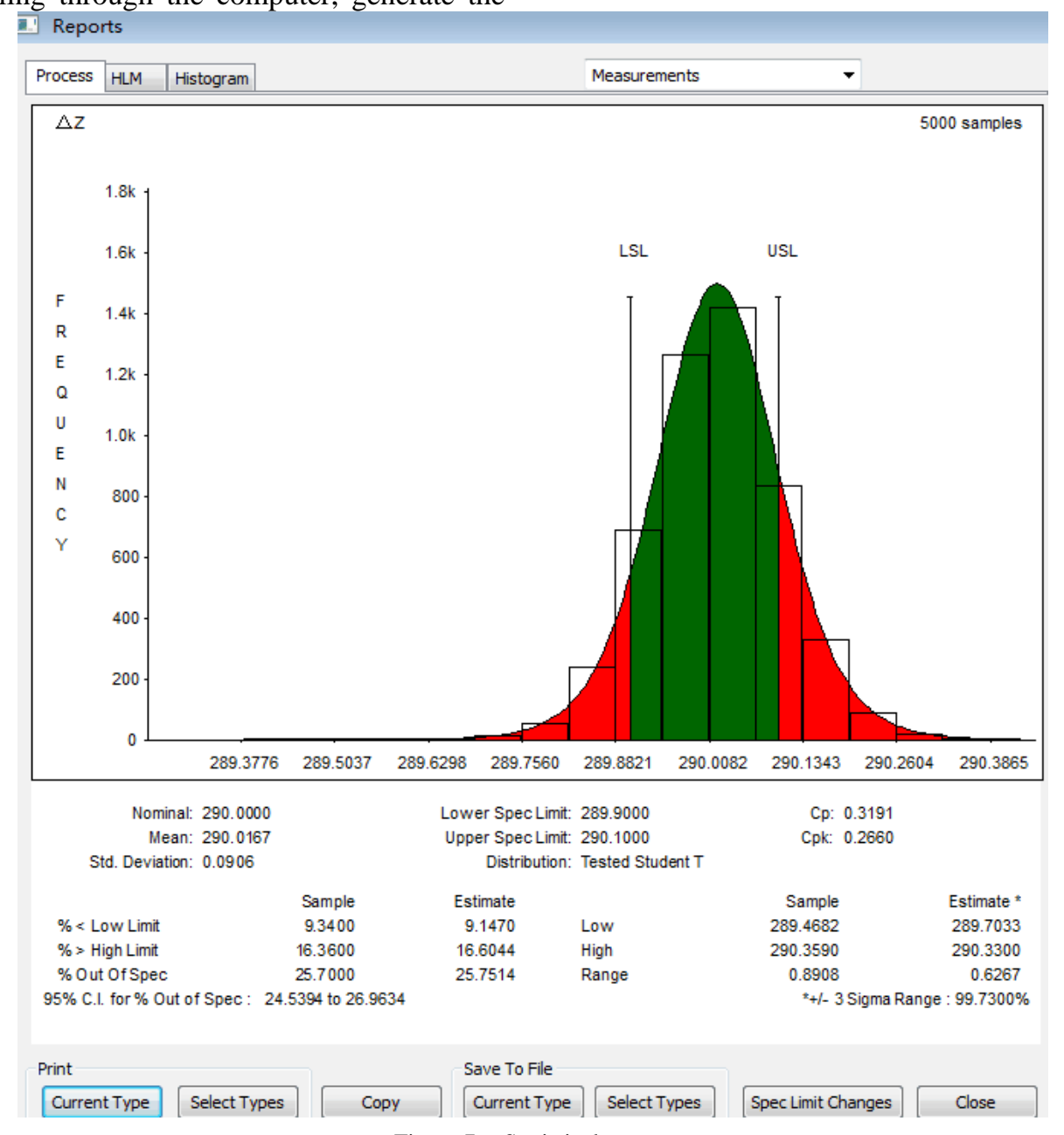

Figure 7. Statistical report. 


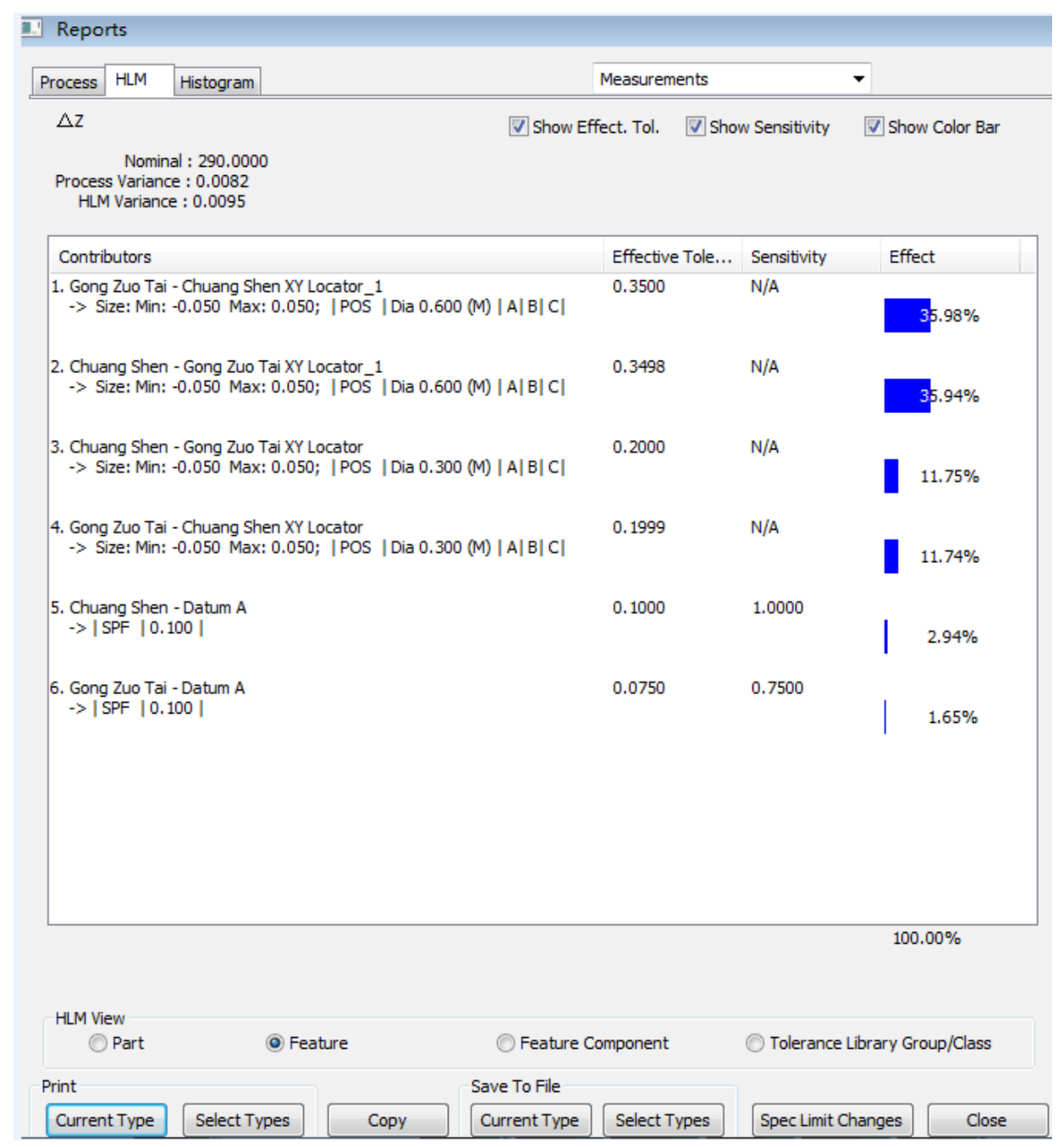

Figure 8. Contribution factor of deviation source.

When use computer aided tolerance analysis software Visvsa to analyze assembly error of five-axis machine tools. We can find out the biggest affects factor of the target size. According to the analysis result, we have a reasonable purpose to modify the corresponding parts of tolerance values. On one hand, it can realize 3D tolerance design and verification based on the demand of comprehensive geometric accuracy of five-axis machine tools, and guarantee comprehensive geometric accuracy of machine tools to specific tolerance of each part of kinematic pair finally. At the same time, we can use contribution factor to provide good basis for subsequent comprehensive error compensation direction of machine tool. On the other hand, we evaluate process processing ability to judge if meet the processing quality and product quality stability according to Cp and Cpk.

\section{CONCLUSION}

From the perspective of improving and guaranteeing comprehensive geometric accuracy of five-axis machine tools, this paper put forward a method of design and verification of five-axis machine tools based on the requirement of comprehensive geometric accuracy. Through the establishment of geometric tolerance analysis model of machine tool, combining with randomness of comprehensive error of machine tool, and using Monte Carlo simulation method for three dimensional error simulation analysis. On one hand, It realize 3D tolerance design and verification based on the requirement of the comprehensive geometric accuracy of five-axis machine tools. To make comprehensive geometric accuracy of machine tools to ensure specific tolerance of each parts of kinematic pair. Through the reasonable contribution factor, it also can modify the corresponding tolerance values of parts. On the other hand, it can provide good evidence for subsequent comprehensive error compensation of five-axis machine tools by using the contribution factor.

\section{REFERENCES}

[1] li Ming FeiLiNa. Coordinate geometry measurement technology and its application [M]. China quality press \& China standard press, 2012.11.

[2] Chiang forward. The new generation GPS standard theory and application. Beijing: higher education press, 2007. 
[3] Lu Jiang, you-liang zhang. Six legs parallel machine tool precision integrated using the monte carlo method [J]. China mechanical engineering, 200213 (6) : 464-467.

[4] Ngoi BKA,Teck O C.A tolerancing optimization method for product design [J]. The International Journal of Advanced Manufacturing Technology,1997,13(4):290-299.

[5] Ma Lynn, huangtian, Wang Yangdeng. Accuracy of parallel machine tool design for manufacturing ([J]. China mechanical engineering, 1999, 10 (10) : 1114-1118.

[6] Sutherland G H,Roth B.Mechanism design: accounting for manufacturing tolerances and costs in function generating problems[J].Journal of Engineering for Industry,1993,(98):283-286.
[7] da-ming liu, zheng Manila apricot. Monte carlo simulation is applied to the assembly tolerance analysis of defective rate guide [J]. Science and engineering, 2006, 2 (4) : 67-82

[8] wen-zheng ding, Zhou Minghu. Precision of multi-axis machine tool design for remanufacturing research [J]. Journal of applied basic and engineering science journal, 2007 (4) : 559-567.

[9] Dufour P., and Groppetti R., Computer Aided Accuracy Improvement in Large NC Machine-Tools [A], MTDR. Conf. Proc. [C], 1981, 22: 611-618.

[10] Autumn, Mo Shuai. Concurrent tolerance optimization design based on improved cost tolerance model [J]. Journal of tianjin university of science and technology, 2010, 25 (5) : 53-56. 